\title{
Detection of Anti-Toxoplasma gondii IgG and IgM Antibodies and Associated Risk Factors during Pregnancy in Southwest Iran
}

\author{
Shahrzad Soltani $\mathbb{D}^{1}$, Ali Dalir Ghaffari $\mathbb{D}^{2}{ }^{2}$ Mehdi Sagha Kahvaz $\left(\mathbb{D},{ }^{1}\right.$ \\ Mohamad Sabaghan $\mathbb{D}^{3},{ }^{3}$ Marzieh Pashmforosh $\mathbb{D}^{3},{ }^{3}$ and Masoud Foroutan $\mathbb{D}^{1}$ \\ ${ }^{1}$ USERN Office, Abadan University of Medical Sciences, Abadan, Iran \\ ${ }^{2}$ Department of Parasitology, Faculty of Medical Sciences, Tarbiat Modares University, Tehran, Iran \\ ${ }^{3}$ Behbahan Faculty of Medical Sciences, Behbahan, Iran
}

Correspondence should be addressed to Marzieh Pashmforosh; marzie_pf@yahoo.com and Masoud Foroutan; masoud_foroutan_rad@yahoo.com

Received 19 February 2021; Accepted 18 May 2021; Published 27 May 2021

Academic Editor: Bryan Larsen

Copyright (c) 2021 Shahrzad Soltani et al. This is an open access article distributed under the Creative Commons Attribution License, which permits unrestricted use, distribution, and reproduction in any medium, provided the original work is properly cited.

\begin{abstract}
Background. This research was aimed at evaluating the seroprevalence of acute and chronic Toxoplasma gondii (T. gondii) infection in pregnant women and related risk factors in southwest Iran. Methods. In this cross-sectional study, eighty-eight pregnant women were included from October 2019 to December 2019. The presence of anti-T. gondii IgM and IgG antibodies was measured using the enzyme-linked immunosorbent assay (ELISA). In addition, a questionnaire consisting of demographic information was completed for each subject. Results. The overall seroprevalence of T. gondii infection was estimated to be 34.09\% (30/88). Of these, $1(1.13 \%)$ and $29(32.95 \%)$ samples were found positive for IgM and IgG, respectively. Regarding the risk factors, the consumption of raw/undercooked meat $(P$ value $=0.007)$ and history of abortion $(P$ value $=0.017)$ were significantly associated with IgG seroprevalence in pregnant women. Conclusion. The results showed that the pregnant women of southwest Iran might be moderately exposed to $T$. gondii. Since the risk of acute $T$. gondii infection in this susceptible group is very important, regular screening tests to diagnose the infection are recommended before pregnancy.
\end{abstract}

\section{Introduction}

Toxoplasma gondii (T. gondii), an obligate intracellular parasite from the phylum Apicomplexa, is a zoonotic protozoan that infects numerous warm-blooded vertebrate species like humans, livestock, birds, and aquatic mammals $[1,2]$. This parasite impresses one-third of the global population, especially in low-income and developing countries $[3,4]$. Overall, T. gondii requires two hosts to complete its environmental life cycle. Cats (family: Felidae) are the final hosts, whereas most warm-blooded animals act as intermediate hosts [1]. The parasite is transmitted mainly through the following routes: intake of raw/undercooked meat infected with tissue cysts, drinking the water or consumption of unwashed vegetables infected with oocysts, and infection from mother to fetus [5].
The high-risk groups for T. gondii infection are immunocompromised individuals such as patients with cancer, HIVpositive people, and organ transplant recipients [4]. Moreover, T. gondii in those seronegative pregnant women who acquire the infection during their pregnancy period may cause serious abnormalities in the fetus $[6,7]$. Pregnant women generally show no signs of this infection throughout pregnancy. The fetus is likely to be subjected to congenital infection upon maternal infection. The intensity of vertical transmission and harm to the embryo depends entirely on the date of the infection occurring during pregnancy. The longer the time elapses throughout pregnancy, the more parasites can invade the fetus. The disorders after congenital transmission include focal necrosis, inflammation, and disorders like brain and eye injuries. In the case of severe infection, there may be other consequences including microcephaly, 
hydrocephalus, mental retardation, and deafness $[8,9]$. In the absence of prenatal screening programs in a specific region, the high percentage of acute $T$. gondii infections in this susceptible group will remain overlooked and left untreated $[9,10]$. To this end, the objective of this research was to evaluate the seroprevalence of $T$. gondii infection in pregnant women and their related risk factors in southwest Iran.

\section{Materials and Methods}

2.1. Study Area. Abadan City is located in the southwest of Iran. This city is bordered to the west by the Arvand waterway and to the east of the Karun River (Arvand Rood) Bahmanshir outlet near the Iran-Iraq border. Now, its population reaches nearly 350,000 people. Summers are dry and intensely hot, with temperature up to $53^{\circ} \mathrm{C}$. Abadan is particularly one of the few warmest crowded places in the world with several storms of sand and dust annually [11].

2.2. Study Population. In this cross-sectional survey, the study population was pregnant women who were referred to the Taleghani and Beheshti hospitals affiliated to the Abadan Faculty of Medical Sciences from October 2019 to December 2019 in the southwest Iran (Abadan County, Khuzestan Province). Accordingly, eighty-eight blood samples were collected from pregnant women. All pregnant women voluntarily agreed to be examined. A written informed consent form was completed and signed by each subject. The study protocol was approved by the Ethical Committee of the Behbahan Faculty of Medical Sciences (IR.BHN.REC.1399.007).

2.3. Questionnaire. A questionnaire consisting of demographic information was completed for each person, including age (10$20,21-30,31-40$, and 41-50), place of residence (urban or rural), education level (diploma or lower and university degree), reason for referral (routine checkup or abortion), contact with cat (yes or no), history of abortion (yes or no), consumption of raw/undercooked meat (yes or no), and source of drinking water (purified or unpurified water).

2.4. Serology. Five milliliters of women's blood samples was taken. All the blood samples were transferred to the central laboratory of the Abadan Faculty of Medical Sciences. First, the blood samples were centrifuged for five minutes at $4000 \mathrm{rpm}$. The sera were then separated and placed at $-20^{\circ} \mathrm{C}$ until analysis. The presence of anti-Toxoplasma IgM and IgG antibodies was evaluated via the enzyme-linked immunosorbent assay (ELISA), with an ELISA kit (Torch-IgG, IgM-Trinity Biotech Company) according to the manufacturer's instructions. The samples with international units (IU) $/ \mathrm{ml}$ of $<0.9,0.9-1.1$, and $>1.1$ were considered negative, borderline, and positive, respectively [11].

2.5. Statistical Analysis. All data were imported into the Statistical Package for the Social Sciences (SPSS) software (version 21) (SPSS Inc., Chicago, IL, USA) for more analysis. For this purpose, the Pearson chi-square and Fisher exact tests were used. The significance level was defined to be less than 0.05 .

\section{Results}

3.1. Seroprevalence of Anti-Toxoplasma Antibodies. In this research, 88 pregnant women were screened by the ELISA method for the presence of anti-Toxoplasma IgG and IgM antibodies. The overall seroprevalence of $T$. gondii infection was $34.09 \%(30 / 88)$. Of these, 1 (1.13\%) and 29 (32.95\%) samples were identified positive for IgM and IgG, respectively (Table 1).

3.2. Risk Factors. Eight risk factors related to T. gondii infection were recorded in this study. In terms of the residence, $30.35 \%(17 / 56)$ and $40.62 \%(13 / 32)$ of the subjects from urban and rural communities were seropositive for $T$. gondii, respectively ( $P$ value $=0.493$ and 0.364 for $\operatorname{IgG}$ and $\operatorname{IgM}$, respectively). All the participants were divided into four categories which include 10-20, 21-30, 31-40, and 41-50 years, and the exposure to T. gondii was estimated as $20 \%(3 / 15)$, $38.09 \%$ (16/42), $34.61 \%(9 / 26)$, and $40 \%(2 / 5)$, respectively ( $P$ value $=0.711$ and 0.775 for IgG and IgM, respectively) In addition, a higher seropositivity was found in participants with a diploma or lower education level $(38.98 \% ; 23 / 59)(P$ value $=0.217$ and 0.481 for IgG and IgM, respectively), in individuals who had direct contact with cats $(41.02 \%$; $16 / 39)(P$ value $=0.327$ and 0.443 for IgG and IgM, respectively), in individuals who drank unpurified water $(52.17 \%$; 12/23) $(P$ value $=0.077$ and 0.75 for IgG and IgM, respectively), and in individuals who were referred due to abortion $(50 \% ; 5 / 10)(P$ value $=0.724$ and 0.898 for $\operatorname{IgG}$ and $\operatorname{IgM}$, respectively). In the above-mentioned risk factors, there was no significant association between the seroprevalence of T. gondii infection and IgG and IgM antibodies, but there was a statistical association between the IgG level and history of abortion $(60 \% ; 12 / 20)(P$ value $=0.017$ and 0.277 for IgG and IgM, respectively) and consumption of raw/undercooked meat $(52.94 \% ; 18 / 34)(P$ value $=0.007$ and 0.386 for IgG and IgM, respectively). Table 1 summarizes the main features and risk factors associated with $T$. gondii prevalence rates in southwest Iran.

\section{Discussion}

Acute T. gondii infection in pregnant women may trigger congenital toxoplasmosis if ignored. The infection can lead to significant and progressive diseases with high morbidity and mortality in fetuses and newborns $[8,9]$. Therefore, in this study, we evaluated the prevalence of specific $T$. gondii IgG and IgM in pregnant women in Abadan City, southwest Iran.

Numerous studies investigated the prevalence of $T$. gondii infection in pregnant women, which differs across regions. For example, findings in the Americas and Africa have shown a higher prevalence, ranging from $6.1 \%$ to $77.5 \%$ and $25.3 \%$ to $75.2 \%$, respectively. Also, in Europe and Asia, the seroprevalence rate is between $9.1-63.2 \%$ and $0.8-60.4 \%$, respectively [12]. As shown in a recent review article with a meta-analysis approach by Torgerson and Mastroiacovo, the annual incidence of congenital toxoplasmosis has been estimated to be 190,100 cases $(179,300-206,300)$ worldwide [10]. In this survey, 88 pregnant women were 
TABLE 1: Demographic characteristics and risk factors related to IgG (latent) and IgM (acute) seroprevalence of T. gondii in pregnant women in Abadan County, Khuzestan Province, Iran, from Oct. to Dec. 2019. $P$ value $<0.05$ indicates a significant difference in seroprevalence between the categories within each characteristic.

\begin{tabular}{|c|c|c|c|c|c|c|}
\hline Characteristic & $\begin{array}{c}\text { Number tested } \\
\text { (percent of total tested) }\end{array}$ & $\begin{array}{c}\text { IgG positive } \\
\text { Number (percent) }\end{array}$ & $P$ value & $\begin{array}{c}\text { IgM positive } \\
\text { Number (percent) }\end{array}$ & $P$ value & $\begin{array}{c}\text { Total } \\
\text { Number (percent) }\end{array}$ \\
\hline Total & $88(100 \%)$ & $29(32.95 \%)$ & & $1(1.13 \%)$ & & $30(34.09 \%)$ \\
\hline \multicolumn{7}{|l|}{ Age } \\
\hline $10-20$ & $15(17.04 \%)$ & $3(20 \%)$ & \multirow{4}{*}{0.711} & $0(0 \%)$ & \multirow{4}{*}{0.775} & $3(20 \%)$ \\
\hline $21-30$ & $42(47.72 \%)$ & $15(35.71 \%)$ & & $1(2.38 \%)$ & & $16(38.09 \%)$ \\
\hline $31-40$ & $26(29.54 \%)$ & $9(34.61 \%)$ & & $0(0 \%)$ & & $9(34.61 \%)$ \\
\hline $41-50$ & $5(5.68 \%)$ & $2(40 \%)$ & & $0(0 \%)$ & & $2(40 \%)$ \\
\hline \multicolumn{7}{|l|}{ Residence } \\
\hline Urban & $56(63.63 \%)$ & $17(30.35 \%)$ & \multirow{2}{*}{0.493} & $0(0 \%)$ & \multirow{2}{*}{0.364} & $17(30.35 \%)$ \\
\hline Rural & $32(36.37 \%)$ & $12(37.50 \%)$ & & $1(3.12 \%)$ & & $13(40.62 \%)$ \\
\hline \multicolumn{7}{|l|}{ Education level } \\
\hline Diploma or lower & $59(67.05 \%)$ & $22(37.28 \%)$ & \multirow{2}{*}{0.217} & $1(1.69 \%)$ & \multirow{2}{*}{0.481} & $23(38.98 \%)$ \\
\hline University degree & $29(32.95 \%)$ & $7(24.13 \%)$ & & $0(0 \%)$ & & $7(24.13 \%)$ \\
\hline \multicolumn{7}{|l|}{ Contact with cat } \\
\hline Yes & $39(44.31 \%)$ & $15(38.46 \%)$ & \multirow{2}{*}{0.327} & $1(2.56 \%)$ & \multirow{2}{*}{0.443} & $16(41.02 \%)$ \\
\hline No & $49(55.69 \%)$ & $14(28.57 \%)$ & & $0(0 \%)$ & & $14(28.57 \%)$ \\
\hline \multicolumn{7}{|c|}{ Consumption of raw/undercooked meat } \\
\hline Yes & $34(38.63 \%)$ & $17(50 \%)$ & \multirow{2}{*}{0.007} & $1(2.94 \%)$ & \multirow{2}{*}{0.386} & $18(52.94 \%)$ \\
\hline No & $54(61.37 \%)$ & $12(22.22 \%)$ & & $0(0 \%)$ & & $12(22.22 \%)$ \\
\hline \multicolumn{7}{|c|}{ Source of drinking water } \\
\hline Purified water & $65(73.87 \%)$ & $18(27.69 \%)$ & \multirow{2}{*}{0.077} & $0(0 \%)$ & \multirow{2}{*}{0.75} & $18(27.69 \%)$ \\
\hline Unpurified water & $23(26.13 \%)$ & $11(47.82 \%)$ & & $1(4.34 \%)$ & & $12(52.17 \%)$ \\
\hline \multicolumn{7}{|l|}{ Reason for referral } \\
\hline Routine checkup & $78(88.63 \%)$ & $25(32.05 \%)$ & \multirow{2}{*}{0.724} & $0(0 \%)$ & \multirow{2}{*}{0.898} & $25(32.05 \%)$ \\
\hline Abortion & $10(11.36 \%)$ & $4(40 \%)$ & & $1(1.13 \%)$ & & $5(50 \%)$ \\
\hline \multicolumn{7}{|l|}{ History of abortion } \\
\hline Yes & $20(22.73 \%)$ & $11(55 \%)$ & \multirow{2}{*}{0.017} & $1(5 \%)$ & \multirow{2}{*}{0.277} & $12(60 \%)$ \\
\hline No & $68(77.27 \%)$ & $18(26.47 \%)$ & & $0(0 \%)$ & & $18(26.47 \%)$ \\
\hline
\end{tabular}

tested for the presence of anti-Toxoplasma IgG and IgM antibodies. The overall seroprevalence of T. gondii infection was 34.09\% (30/88) in Abadan City. In two different global metaanalysis studies, the seroprevalence of latent and acute $T$. gondii infection in pregnant women was estimated to be $33.8 \%$ (95\% CI: 31.8-35.9\%) and 1.1\% (95\% CI: 0.9-1.2\%), respectively $[3,9]$. In another report by Foroutan-Rad et al., the pooled seroprevalence of $T$. gondii infection among the Iranian pregnant women was $41 \%(95 \% \mathrm{CI}=36-45 \%)$ [13]. Thus, the seroprevalence rate of the current study was comparable with the Iranian pregnant women's average seroprevalence. In a study by Akhlaghi et al. performed on 400 pregnant women in Karaj, the presence of anti-Toxoplasma IgG and IgM antibodies was $29 \%$ and $1 \%$, respectively [14]. In Khorramabad [15], the seroprevalence rate of $T$. gondii infection in pregnant women was estimated to be $31 \%$, which is in accordance with the current study, whereas the seroprevalence rate in pregnant women in Kermanshah [16], Isfahan [17], and Sabzevar [18] was 22.7\%, 20.1\%, and $19.2 \%$, respectively, which is lower than the results of the current study.
There are several probable risk factors for acquiring the T. gondii infection, although there are abundant inconsistent outcomes. In this study, a questionnaire was used to document eight relevant risk factors associated with $T$. gondii infection in pregnant women. Upon analysis, we found a statistically significant association between IgG seroprevalence and raw/undercooked meat intake $(P$ value $=0.007)$ and history of abortion $(P$ value $=0.017)$. The other risk factors showed no significant association. Intake of raw/undercooked meat is often seen as a major risk factor for the infection [19]. Consumption of raw or half-cooked meat has been reported as one of the most significant sources of human infection [20]. According to Jones et al.'s study, working with meat and consuming undercooked meat are two of the most significant risk factors for $T$. gondii infection in the United States [21]. The findings of the current study demonstrated a meaningful association between the consumption of raw or half-cooked meat and $T$. gondii infection $(P$ value $=$ $0.007)$. In line with our study, some studies believed that eating raw/undercooked meat could significantly increase the infection rates in societies $[22,23]$. For example, in a study 
by Khademi et al. in Hormozgan Province (southern Iran), a significant association was observed between the consumption of raw/semi-raw meat and T. gondii infection [24]. In contrast to our research, there was no statistically different between the consumption of raw/semi-raw meat and toxoplasmosis in surveys conducted by Ertug et al. [25] and Eshratkhah et al. [20]. A study by Rasti et al. in 2017 showed that the prevalence of $T$. gondii infection in sheep and goats ranges from 3.3 to $38 \%$ in various parts of Iran. In addition, they showed that sheep and goat are important reservoirs of T. gondii [26]. Therefore, the researchers revealed the role of meat and other meat products as sources for T. gondii.

In this study, a significant association between T. gondii and the history of abortion was observed $(P$ value $=0.017)$. Similar to our study, in studies by Hosseinzadeh et al. [27], Aali et al. [28], and Sahwi et al. [29], the seroprevalence of T. gondii $\operatorname{IgG}$ and IgM in pregnant women with a history of abortion was statistically higher than that in control groups, which indicates a potential association between $T$. gondii infection and the abortion. However, in contrast with our study, some studies such as Akhlaghi et al. [14], Eshratkhah et al. [20], Babaie et al. [30], and Hajsoleimani et al. [31] did not show any meaningful association between toxoplasmosis and history of abortion. Also, in a study by Ghasemi et al. which evaluated the role of toxoplasmosis in etiology of abortion and stillbirth, the results showed that the IgG seroprevalence was $25.5 \%$ in the case group $(26.8 \%$ in abortion and $21.4 \%$ in stillbirth), and no statistically significant differences were seen in the case and control groups [32].

To assess T. gondii seroprevalence among various age groups, the participants were categorized into four groups, including 10-20, 21-30, 31-40, and 41-50 years. The seroprevalence rate was $20 \%, 38.09 \%, 34.61 \%$, and $40 \%$ in these groups, respectively. Although there was no significant association between $T$. gondii infection and different age groups in the current study ( $P=0.711$ and 0.775 for IgG and IgM, respectively), the seroprevalence rate in 41-50 years was higher than that in other groups, which is in accordance with Alvarado-Esquivel et al. [33], Fallah et al. [34], and Daryani and Sagha [35] studies. The seroprevalence rate tended to increase with age. The cause for this may be the rising risk of exposure with age. In a meta-analysis study by Borna et al., in line with our study, the seroprevalence of T. gondii IgG increased with age [36].

In the current research, T. gondii infection in women who had a history of contact with cats was higher, but the findings were not significant. Nonetheless, a previously published study among the general Iranian population $(P<0.05)$ suggested that contact with cat significantly correlates with T. gondii infection [22]. Cats are the only definitive host for the parasites, and due to their close contact with people, especially in the rural areas, there is a general opinion that contact with the cat could be a major risk factor for acquiring the infection $[1,37]$.

In the current study, $30.35 \%$ and $40.62 \%$ of pregnant women from urban and rural regions were seropositive for T. gondii infection, respectively. Villagers' lifestyles such as direct contact with soil, livestock, and animals could make this situation predictable, as previously reported in most of the published articles $[13,38]$. Risk factor assessment in the general Iranian population has shown that $T$. gondii infection can be reduced by growing schooling grades $(P<0.0001)$ [22]. In this study, the same association was observed, but no significant association was observed between $T$. gondii infection and education level. As it is evident, lack of understanding of the primary transmission pathways and sources of infection as well as sanitary conditions are remarkably significant factors for T. gondii infection.

This research estimated that the seroprevalence of acute T. gondii infection in pregnant women was $1.13 \%(1 / 88)$. In Iran, the seroprevalence of acute infection during the pregnancy was reported to be $1.4 \%$ in Zanjan [31], $4.8 \%$ in Isfahan [39], 0.5\% in Urmia [20], and 0.6\% in Kashan [40]. The reason for different infection rates in various areas of Iran and the world may be related to socio-cultural conditions, contact with cats, and observance of health points [41].

\section{Limitations}

Lack of supporting serological data by molecular confirmation and the low number of sample size are the limitations of the study.

\section{Conclusions}

In conclusion, our study revealed an overall seroprevalence of $34.09 \%$ with T. gondii infection in pregnant women in Abadan City (southwest of Iran). Because the risk of acute T. gondii infection in this susceptible group is very important, enhanced control and prevention attempts should be rigorously carried out. In addition, regular screening tests for $T$. gondii infection could also be useful in the routine clinical care of pregnant women. Eventually, improving women's awareness regarding toxoplasmosis and its consequences, the main transmission routes, and associated risk factors can surely decrease the rate of seroprevalence, particularly in rural communities. Health officials should regard the screening test for T. gondii infection before marriage or pregnancy as a routine test.

\section{Abbreviations}

ELISA: Enzyme-linked immunosorbent assay

IgG: Immunoglobulin G

IgM: Immunoglobulin M

T. gondii: Toxoplasma gondii.

\section{Data Availability}

The data used to support the findings of this study are available from the corresponding author upon reasonable request.

\section{Ethical Approval}

All subjects voluntarily agreed to participate. Written informed consent was obtained from all the subjects. This study received the approval from the Behbahan Faculty of Medical Sciences Ethical Committee (IR.BHN.REC.1399.007). 


\section{Disclosure}

The funders of this study had no role in the study design, the analysis and interpretation of data, the writing of the final paper, and the decision to submit the manuscript for publication.

\section{Conflicts of Interest}

The authors declared no potential conflicts of interest with respect to the research, authorship, and/or publication of this article.

\section{Authors' Contributions}

SS and MF designed the study protocol. SS, MS, MSK, MP, and MF collected the data and were involved in statistical analysis. SS performed the experiments. ADG drafted the manuscript. SS and MF critically revised the manuscript. All authors read and approved the final version of the manuscript. The corresponding authors had access to the data in the study and had the final responsibility for the decision to submit for publication.

\section{Acknowledgments}

The authors sincerely appreciate all personnel of Taleghani and Beheshti hospitals in Abadan City for their kind cooperation. We are very grateful to Dr. Sanaz Tavakoli (https:// orcid.org/0000-0002-3837-1961) (Department of Parasitology and Mycology, School of Medicine, Isfahan University of Medical Sciences, Isfahan, Iran) and Mrs. Fatemeh Maghsoudi (Abadan Faculty of Medical Sciences, Abadan, Iran) for their helpful consultation and comments on the manuscript. This study was financially supported by Grant No. 98113 from the Behbahan Faculty of Medical Sciences, Behbahan, Iran.

\section{References}

[1] J. P. Dubey, "The history of Toxoplasma gondiiâ-the first 100 years," The Journal of Eukaryotic Microbiology, vol. 55, no. 6, pp. 467-475, 2008.

[2] M. Foroutan, Y. Fakhri, S. M. Riahi et al., "The global seroprevalence of Toxoplasma gondii in pigs: a systematic review and meta-analysis," Veterinary Parasitology, vol. 269, pp. 42-52, 2019.

[3] A. Rostami, S. M. Riahi, H. R. Gamble et al., "Global prevalence of latent toxoplasmosis in pregnant women: a systematic review and meta-analysis," Clinical Microbiology and Infection, vol. 26, no. 6, pp. 673-683, 2020.

[4] Z. D. Wang, H. H. Liu, Z. X. Ma et al., "Toxoplasma gondii infection in immunocompromised patients: a systematic review and meta-analysis," Frontiers in Microbiology, vol. 8, p. 389,2017

[5] F. Robert-Gangneux and M. L. Darde, "Epidemiology of and diagnostic strategies for toxoplasmosis," Clinical Microbiology Reviews, vol. 25, no. 2, pp. 264-296, 2012.

[6] S. Fallahi, A. Rostami, M. N. Shiadeh, H. Behniafar, and S. Paktinat, "An updated literature review on maternal-fetal and reproductive disorders of _Toxoplasma gondii_ infec- tion," Journal of gynecology obstetrics and human reproduction, vol. 47, no. 3, pp. 133-140, 2018.

[7] S. Rasti, F. S. Ghasemi, A. Abdoli, A. Piroozmand, S. G. A. Mousavi, and Z. Fakhrie-Kashan, "ToRCH "co-infections" are associated with increased risk of abortion in pregnant women," Congenital Anomalies, vol. 56, no. 2, pp. 73-78, 2016.

[8] E. J. Goldstein, J. G. Montoya, and J. S. Remington, "Management of Toxoplasma gondii infection during pregnancy," Clinical Infectious Diseases, vol. 47, pp. 554-566, 2008.

[9] A. Rostami, S. M. Riahi, D. G. Contopoulos-Ioannidis et al., "Acute Toxoplasma infection in pregnant women worldwide: a systematic review and meta-analysis," PLoS Neglected Tropical Diseases, vol. 13, no. 10, article e0007807, 2019.

[10] P. R. Torgerson and P. Mastroiacovo, "The global burden of congenital toxoplasmosis: a systematic review," Bulletin of the World Health Organization, vol. 91, no. 7, pp. 501-508, 2013.

[11] S. Soltani, M. Foroutan, H. Afshari, M. Hezarian, and M. S. Kahvaz, "Seroepidemiological evaluation of Toxoplasma gondii immunity among the general population in southwest of Iran," Journal of parasitic diseases, vol. 42, no. 4, pp. 636642, 2018.

[12] G. Pappas, N. Roussos, and M. E. Falagas, “Toxoplasmosis snapshots: global status of Toxoplasma gondii seroprevalence and implications for pregnancy and congenital toxoplasmosis," International Journal for Parasitology, vol. 39, no. 12, pp. 1385-1394, 2009.

[13] M. Foroutan-Rad, S. Khademvatan, H. Majidiani, S. Aryamand, F. Rahim, and A. S. Malehi, "Seroprevalence of Toxoplasma gondii in the Iranian pregnant women: a systematic review and meta-analysis," Acta Tropica, vol. 158, pp. 160-169, 2016.

[14] L. Akhlaghi, A. Ghasemi, R. Hadighi, and F. Tabatabaie, "Study of seroprevalence and risk factors for Toxoplasma gondii among pregnant women in Karaj township of Alborz province [2013]," Journal of Entomology and Zoology Studies, vol. 2, pp. 217-219, 2014.

[15] K. Cheraghipour, H. Taherkhani, M. Fallah et al., "Seroprevalence of toxoplasmosis in pregnant women admitted to the health centers of Khorram-Abad City, Iran," Avicenna Journal of Clinical Medicine, vol. 17, pp. 46-51, 2010.

[16] A. Athari, S. Shojaeian, O. Eliasi, and K. Delfani, "Seroprevalence of toxoplasma antibodies among pregnant women in Kerman Shah," Medical Journal of The Islamic Republic of Iran (MJIRI), vol. 8, pp. 93-95, 1994.

[17] T. Jalayer and T. Alame, "Seroprevalence of congenital toxoplasmosis in neonatal born in Isfahan," Second congress of parasitology in Tehran (Iran), vol. 23, Iranian Journal of Parasitology, 1997.

[18] H. Moalaee, E. Shirzad, and M. Namazi, "Seroepidemiology of toxoplasmosis and its eye complication in pregnant women," Sabzavar University Medical Sciences Journal (Iran), pp. 2123, 1992.

[19] S. Belluco, M. Mancin, D. Conficoni, G. Simonato, M. Pietrobelli, and A. Ricci, "Investigating the determinants of Toxoplasma gondii prevalence in meat: a systematic review and meta-regression," PLoS One, vol. 11, no. 4, p. e0153856, 2016.

[20] A. Eshratkhah Mohammadnejad, G. Eslami, F. Shamsi et al., "Prevalence of food-borne Toxoplasma in pregnant women population of Urmia, Iran," Journal of food quality and hazards control, vol. 5, pp. 17-23, 2018. 
[21] J. L. Jones, V. Dargelas, J. Roberts, C. Press, J. S. Remington, and J. G. Montoya, "Risk factors for Toxoplasma gondii infection in the United States," Clinical Infectious Diseases, vol. 49, no. 6, pp. 878-884, 2009.

[22] A. Daryani, S. Sarvi, M. Aarabi et al., "Seroprevalence of Toxoplasma gondii in the Iranian general population: a systematic review and meta-analysis," Acta Tropica, vol. 137, pp. 185194, 2014.

[23] M. Foroutan-Rad, H. Majidiani, S. Dalvand et al., "Toxoplasmosis in blood donors: a systematic review and meta-analysis," Transfusion Medicine Reviews, vol. 30, no. 3, pp. 116-122, 2016.

[24] S. Z. Khademi, F. Ghaffarifar, A. Dalimi, P. Davoodian, and A. Abdoli, "Prevalence and risk factors of Toxoplasma gondii infection among pregnant women in Hormozgan Province, south of Iran," Iranian Journal of Parasitology, vol. 14, p. 167, 2019.

[25] S. Ertug, P. Okyay, M. Turkmen, and H. Yuksel, "Seroprevalence and risk factors for toxoplasmainfection among pregnant women in Aydin Province, Turkey," BMC public health, vol. 5, no. 1, 2005.

[26] S. Rasti, N. Marandi, A. Abdoli, M. Delavari, and S. G. A. Mousavi, "Serological and molecular detection ofToxoplasma gondiiin sheep and goats in Kashan, Central Iran," Journal of food safety, vol. 38, no. 2, 2018.

[27] M. Hosseinzadeh, A. Khosravi, L. Shidelzadeh, B. Kalantari, and R. Ranjbar, "Seroepidemiological assessment of Toxoplasmosis in women with and without abortion in Ilam," Health System Research, vol. 6, pp. 626-634, 2011.

[28] B. S. Aali, F. Harandi, E. Nazari, and Z. Salari, "Comparison of Toxoplasma gondii IgG and IgM seropositivity between women with spontaneous abortions and ongoing pregnancies," The Iranian Journal of Obstetrics, Gynecology and Infertility, vol. 14, pp. 1-6, 2011.

[29] S. Y. Sahwi, M. S. Zaki, N. Y. Haiba, O. K. Elsaid, M. Y. Anwar, and S. A. AbdRabbo, "Toxoplasmosis as a cause of repeated abortion," Journal of Obstetrics and Gynaecology, vol. 21, no. 2, pp. 145-148, 1995.

[30] J. Babaie, S. Amiri, E. Mostafavi et al., "Seroprevalence and risk factors for Toxoplasma gondii infection among pregnant women in Northeast Iran," Clinical and Vaccine Immunology, vol. 20, no. 11, pp. 1771-1773, 2013.

[31] F. Hajsoleimani, A. Ataeian, A. Nourian, and S. Mazloomzadeh, "Seroprevalence of Toxoplasma gondii in pregnant women and bioassay of IgM positive cases in Zanjan," Northwest of Iran. Iranian journal of parasitology, vol. 7, p. 82, 2012.

[32] F. S. Ghasemi, S. Rasti, A. Piroozmand et al., "Toxoplasmosisassociated abortion and stillbirth in Tehran, Iran," The Journal of Maternal-Fetal \& Neonatal Medicine, vol. 29, no. 2, pp. 248251, 2016.

[33] C. Alvarado-Esquivel, A. Torres-Castorena, O. Liesenfeld et al., "Seroepidemiology of Toxoplasma gondii infection in pregnant women in rural Durango, Mexico," Journal of Parasitology, vol. 95, no. 2, pp. 271-274, 2009.

[34] E. Fallah, R. Navazesh, J. Majidi, H. Kushavar, and N. Mahdipourzareh, "An epidemiological study of toxoplasma infection among high-school girls in Jolfa," Journal of Reproduction \& Infertility, vol. 6, 2005.

[35] A. Daryani and M. Sagha, "Seroepidemiology of toxoplasmosis in women referred to medical health laboratory before mar- riage, Ardebil," Journal of Ardabil University of Medical Sciences, vol. 4, p. 6, 2004.

[36] S. Borna, M. Shariat, M. Fallahi, and L. Janani, "Prevalence of immunity to toxoplasmosis among Iranian childbearing age women: systematic review and meta-analysis," Iranian Journal of Reproductive Medicine, vol. 11, 2013.

[37] M. Foroutan, S. Dalvand, A. Daryani et al., "Rolling up the pieces of a puzzle: a systematic review and meta-analysis of the prevalence of toxoplasmosis in Iran," Alexandria Journal of Medicine, vol. 54, no. 3, pp. 189-196, 2018.

[38] E. Z. Gebremedhin and G. Tadesse, "A meta-analysis of the prevalence of Toxoplasma gondii in animals and humans in Ethiopia," Parasites \& Vectors, vol. 8, no. 1, 2015.

[39] T. Alameh and F. Tavangar, "Frequency of congenital toxoplasmosis and early neonatal morbidity in Shahid Beheshty medical center in Isfahan," Iranian Journal of Obstetrics, Gynecology and Infertility, vol. 5, pp. 6-13, 2002.

[40] S. Rasti, M. Behrashi, M. Bandepour, A. Talebian, and G. Moosavi, "W047 incidence of toxoplasmosis in neonates and its complications," International Journal of Gynecology \& Obstetrics, vol. 119, 2012.

[41] S. A. Chaudhry, N. Gad, and G. Koren, "Toxoplasmosis and pregnancy," Canadian Family Physician, vol. 60, no. 4, pp. 334-336, 2014. 